\title{
Clinical study regarding nasal reconstruction methods
}

\section{Sabina Ionita, Ioan Lascar}

Department of Plastic and Reconstructive Surgery, Emergency Clinical Hospital, Bucharest, Romania

\section{ABSTRACT}

BACKGROUND. In present days, there are described many reconstruction techniques for nasal post-traumatic and post-excision total defects, from simple suture to microsurgical free transfer in nasal reconstruction. It is important to follow the defect dimensions, its topography and also its etiology. The nasal aesthetic subunits principle is very important in aesthetic and functional reconstruction of a nasal defect.

MATERIAL AND METHODS. The clinical study from this paper was realised on the patients admitted in the Department of Plastic and Reconstructive Surgery of the Emergency Clinical Hospital from Bucharest, between 2012 and 2013. Different determinant factors were studied for the success of nasal reconstruction. It was compared the difference between the reconstruction of the whole nasal subunit, based on aesthetic subunits principle, and the reconstruction of the defect.

RESULTS. Although in our clinic the reconstruction of the defect is used in the majority of the cases and rarely the reconstruction based on aesthetic subunits principle, very good results were obtained, with a reduced number of complication and very few relapses.

CONCLUSION. The success of the reconstruction depends on multiple factors regarding the type of the defect. The grafts can give very good results in all aesthetic subunits, while the nasogenian flap has good results for the reconstruction of the nasal wing and lobule defects. The frontal flap is the first option for reconstruction of the dorsum nasi with satisfactory results.

This paper is supported by the Sectoral Operational Programme Human Resources Development (SOP HRD), financed from the European Social Fund and by the Romanian Government under the contract number POSDRU/159/1.5/137390

KEYWORDS: nasal reconstruction, aesthetic subunits, grafts, flaps.

\section{INTRODUCTION}

In present days, many reconstruction techniques for nasal post-traumatic and post-excision defects are described. The nasal pathology is vast, involving traumatology, and also a continual growth of oncological pathology, the incidence of basocellular and squamocellular carcinoma being very high.

There are described many reconstructive techniques from simple suture to microsurgical free transfer in nasal reconstruction after total defects. It is important to follow the defect dimensions, its topography and also its etiology. The nasal aesthetic subunits principle is very important in aesthetic and functional reconstruction of a nasal defect. Modern techniques used in nasal reconstruction are based on this principle of nasal aes- thetic subunits and on the maximisation of the aesthetic and functional results, with a lowering of the number of reinterventions, complications and costs.

There is a controversy in the literature between the defect reconstruction and that of the whole aesthetic subunit. These principles must be customised regarding each patient, in both cases being many favourable studies $^{1}$.

It is preferred that all nasal elements be rebuild in the same surgical intervention: mucosa, skeletal support and the teguments. The reconstruction can be done in different steps, in the first step the nasal mucosa and the bone skeletal support reconstruction being achieved and in the next steps the reconstruction of the external envelope, the wings contour and of the nose tip ${ }^{1}$. 
It is necessary to observe that in the case of small and medium defects it is not important to achieve the aesthetic subunits principle, but in the big defects it must be accomplished ${ }^{2}$.

The nasal aesthetic subunits principle is based on the fact that if an aesthetic subunit presents a defect of $50 \%$ or more, all subunit must be excised. The disadvantage of this procedure is that a lot of healthy tissue is resected. Rohrich stipulates that a satisfactory scar can be placed in the limits of a subunit and one can give up to the destruction of a normal tissue ${ }^{2}$.

This principle was described for the first time by Burget and Menick in 1985. They described the 9 nasal topographical aesthetic subunits: dorsum, tip, pair wings, columella, lateral walls and soft triangles. The incisions will be made at the subunits borders edge for hiding the scars. But this principle is not enough for achieving a satisfactory nasal reconstruction. The colour of the skin, texture, contour and its reflection in the sun must be taken into account ${ }^{3,4}$. It is very important to achieve the nasal symmetry, even if the nasal subunit principles are not applied, the reconstruction of the defect gives better aesthetic and functional results of the donor site and of the defect ${ }^{5}$. The local flaps, as the bilobed flap (Zitelli) and the advancement dorsal rotation flap (Riger), pass the nasal subunits borders, but confer an ideal colour and texture to the skin, which are very important in nasal reconstruction ${ }^{3}$.

Rohrich describes the basic principles for a successful nasal reconstruction: maximal preservation of healthy tissue, defect reconstruction and not the one of the whole unit, complementary ablative procedures as primary dermabrasion, which improves the final results and lowers the number of revision procedures, and, as many times as possible, he refers to the utilisation of axillary flaps, the principal aesthetic objective being to obtain a good contour ${ }^{4}$.

The complications that can occur after nasal reconstruction are infection, necrosis, haematoma, wound contraction and the formation of a vicious scar ${ }^{2}$.

The objective of the present study was to demonstrate if the defect reconstruction is better than the reconstruction of the whole nasal subunit, without affecting in this way other unaffected nasal surfaces.

\section{MATERIAL AND METHODS}

This study was performed on 110 patients admitted in the Department of Plastic and Reconstructive Surgery of the Emergency Clinical Hospital from Bucharest, between 2012 and 2013. There were 51 women $(46.36 \%)$ and 59 men $(53.63 \%)$, with ages between 16 and 93 years (average of 53 years). The num- ber of days of hospitalization varied between 1 and 32 days, with an average of 7 days.

18 factors involved in the success of nasal surgical reconstruction and in the minimalisation of relapse were analysed. It was compared the difference between the reconstruction of the whole nasal subunit, based on aesthetic subunits principle, and the reconstruction of the defect only.

The results were statistically analysed using simple variance analysis (ANOVA test) at a transgression probability of $\mathrm{p}<0.05 \%$. Also, simple correlations between the studied factors were done and the significant correlations between them were established. The determination of the significance between groups was done using Tukey's post hoc test.

\section{RESULTS}

The clinical study on post-traumatic and post-excision nasal defects patients analysed 18 factors and the incidence of each of them was determined: sex, age, days of hospitalization, type of reconstruction, fracture reduction, type of defect, complications, simultaneous diseases, placement of the defect, depth of the defect, treatment, relapse, associated wounds, leukocytes, anemia, total proteins, coagulation modifications and blood sugar levels.

Variance analysis (ANOVA test) of the type of reconstruction factor showed that it depended significantly on the type of defect and its placement, and also on the simultaneous wounds. Moreover, the complications and relapses risk depended significantly on the reconstruction type (Table 1).

The "hospitalization days" factor analysis revealed a statistically significant dependence between the number of hospitalization days and the type of reconstruction used. Also, the number of hospitalization days presented a high dependency on the complications (Table 2).

Simple correlations were done between all the studied factors and we observed the existence of a significant and positive correlation between the type of reconstruction and the number of hospitalization days, placement of the defect and simultaneous wounds. Between the type of reconstruction and the relapse existed a significant and negative correlation. There were also other significant correlations, but without clinical significance.

We observed that the defects found in the patients included in this clinical study were placed in almost all nasal regions, mostly the nasal wing, the dorsum nasi and the nasal pyramid (Figure 1).

For the defects repairing 14 types of reconstruction were used, the most frequent ones being the primary suture and the nasogenian flap (Figure 2). 


\section{Table 1}

Variance analysis (ANOVA test) of the type of reconstruction factor

\begin{tabular}{|c|c|c|c|}
\hline Factor & df & $\mathbf{F}$ & $\mathbf{P}$ \\
\hline Fracture reduction & 12 & 1.48 & 0.141 \\
\hline Type of defect & 12 & 1.86 & $0.048^{*}$ \\
\hline Complications & 12 & 1.65 & $0.090^{*}$ \\
\hline Associated diseases & 12 & 0.48 & 0.919 \\
\hline Place of the defect & 12 & 1.67 & $0.084^{*}$ \\
\hline Depth of the defect & 12 & 2.08 & $0.024^{*}$ \\
\hline Treatment & 12 & 0.67 & 0.767 \\
\hline Relapse & 12 & 3.78 & $0.000^{* * *}$ \\
\hline Associated wounds & 12 & 2.26 & $0.013^{*}$ \\
\hline Leukocyte & 12 & 0.69 & 0.757 \\
\hline Anemia & 12 & 0.87 & 0.572 \\
\hline Total proteins & 12 & 0.42 & 0.950 \\
\hline Coagulation modifications & 12 & 1.78 & $0.061^{*}$ \\
\hline Blood sugar level & 12 & 0.63 & 0.805 \\
\hline
\end{tabular}

Level of stastistical significance: ${ }^{*}$ - significant; ${ }^{* *}$ - very significant; ${ }^{* * *}$ - distinctly significant

\section{Table 2}

Variance analysis (ANOVA test) for the "hospitalization days" factor

\begin{tabular}{|c|c|c|c|}
\hline Factor & df & $\mathbf{F}$ & $\mathbf{P}$ \\
\hline Type of reconstruction & 22 & 3.41 & $0.000^{* * *}$ \\
\hline Fracture reduction & 22 & 0.76 & 0.762 \\
\hline Type of defect & 22 & 0.69 & 0.835 \\
\hline Complication & 22 & 2.69 & $0.000^{* * *}$ \\
\hline Associated diseases & 22 & 1.11 & 0.347 \\
\hline Placement of the defect & 22 & 0.85 & 0.651 \\
\hline Depth of the defect & 22 & 1.48 & 0.100 \\
\hline Treatment & 22 & 0.77 & 0.744 \\
\hline Relapse & 22 & 1.15 & 0.309 \\
\hline Associated wounds & 22 & 0.83 & 0.681 \\
\hline Leukocyte & 22 & 0.80 & 0.711 \\
\hline Anemia & 22 & 1.44 & 0.114 \\
\hline Total proteins & 22 & 0.60 & 0.912 \\
\hline Coagulation modifications & 22 & 1.56 & 0.074 \\
\hline Blood sugar level & 22 & 1.18 & 0.282 \\
\hline
\end{tabular}

Level of stastistical significance: ${ }^{* * *}$ - distinctly significant 


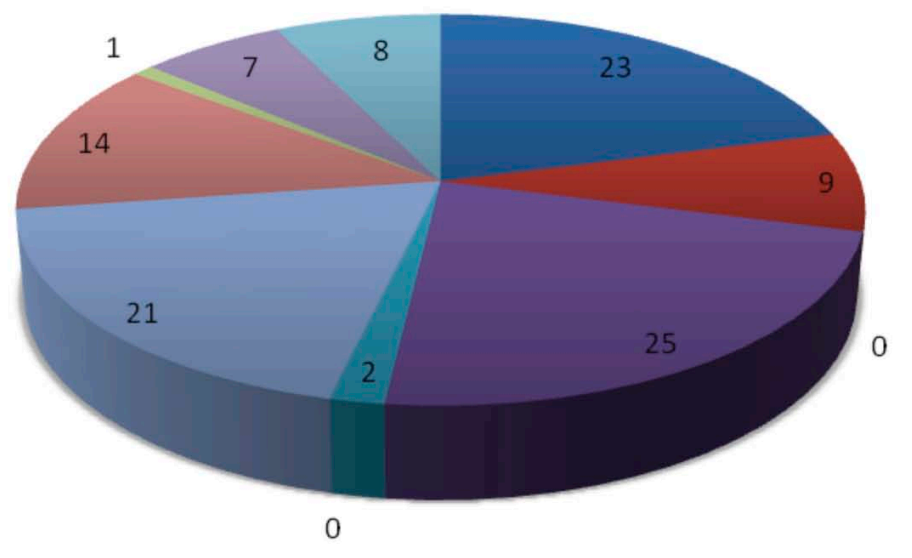

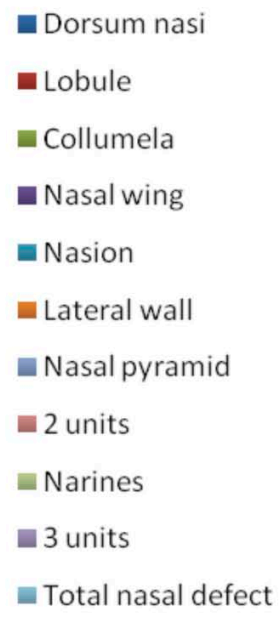

Figure 1. The placement of the defect in the study group

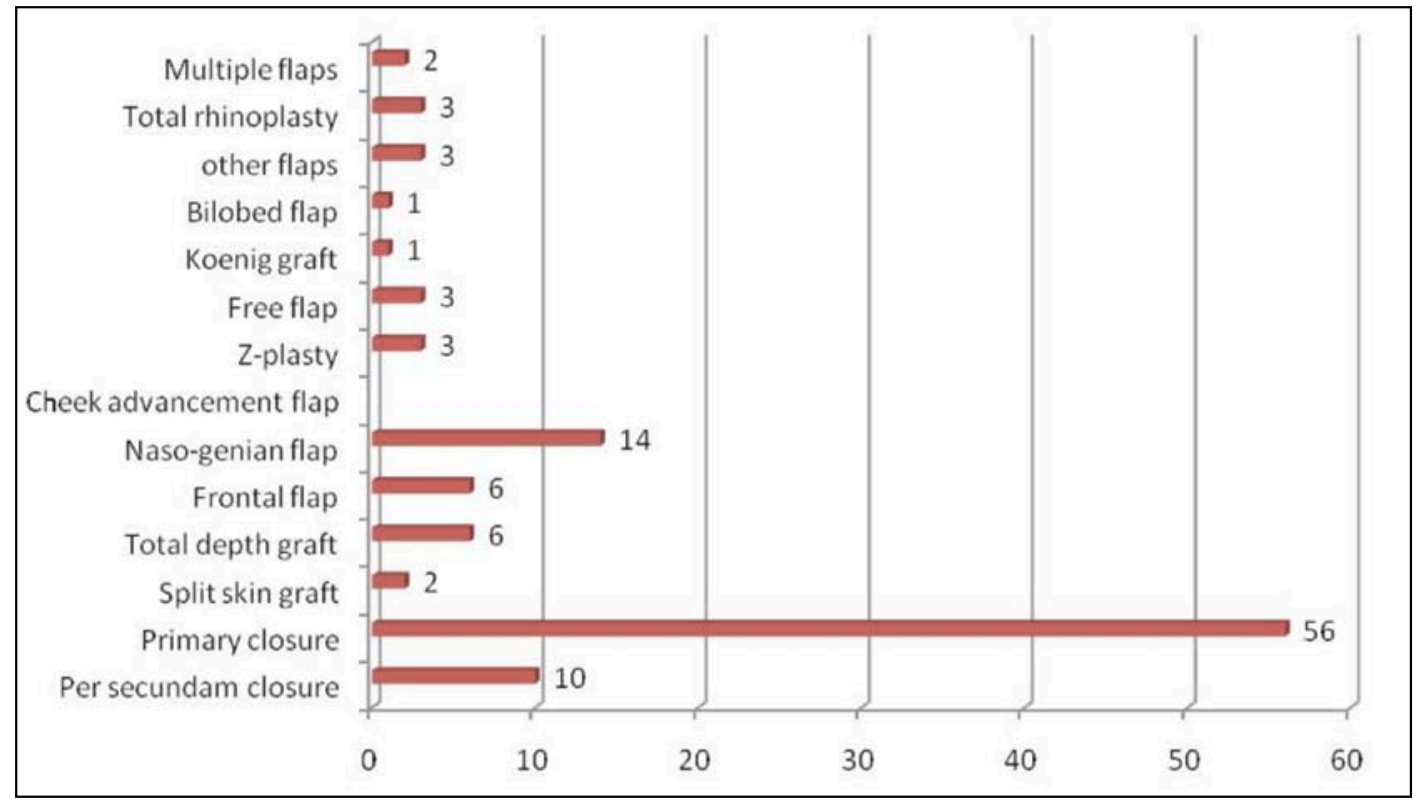

Figure 2. Types of reconstruction used in the study group

6 types of defects were observed, the most frequent being the post-traumatic ones; from the post-excision ones, the most frequent were basocellular carcinoma, this being also the most frequent neoplasm identified (Figure 3).

As regarding the depth of the defect, the most frequently were affected the tegument and subcutaneous tissue, followed by the nasal bones, the cartilage being affected in a lower proportion (only 7\%) (Figure 4).

Analysing the type of reconstruction used regarding the placement of the defect, we observed that, for the nasion, the most frequently was used the closing per secundam. For dorsum nasi, nasal wing, nasal pyramid, nostril, in the case of involvement of three nasal units or of the whole nose, the primary suture was used. For the lobule, primary suture and split skin grafts were performed in the same number of cases. The nasogenian flap was also used for the reconstruction of this nasal region. In those cases with three nasal regions involvement, nasogenian flap and total rhinoplasty were the techniques of choice (Figure 5).

The grafts were used in all aesthetic subunits with very good results, including the defects that involved three subunits.

For the nasal wing defects, the nasogenian flap was mostly used; this technique respects the basic principle of nasal reconstruction, as well as for the lobule reconstruction. For the reconstruction of the dorsum nasi, the frontal flap was the chosen technique (Figure 6). 


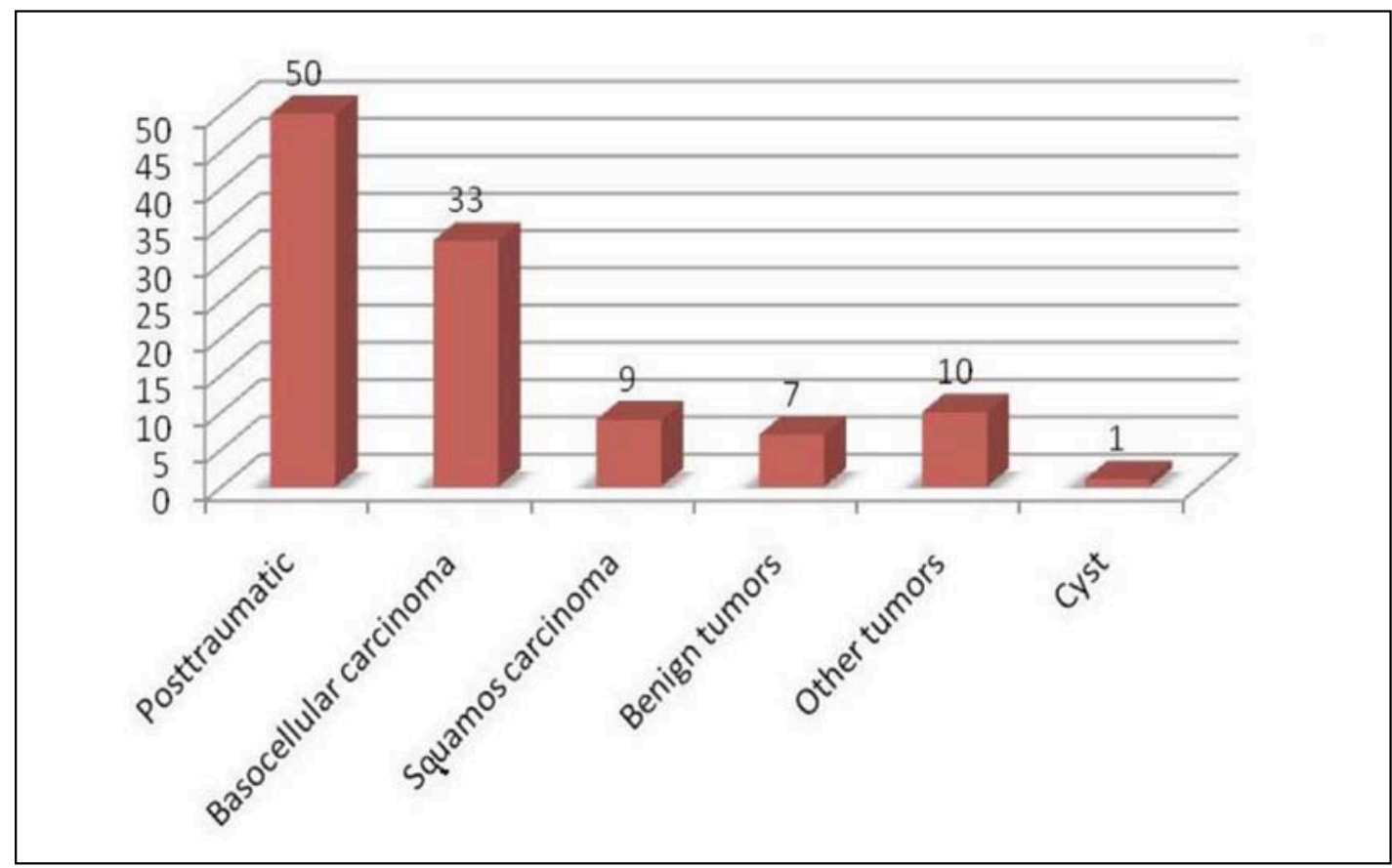

Figure 3. Types of defects observed in the study group

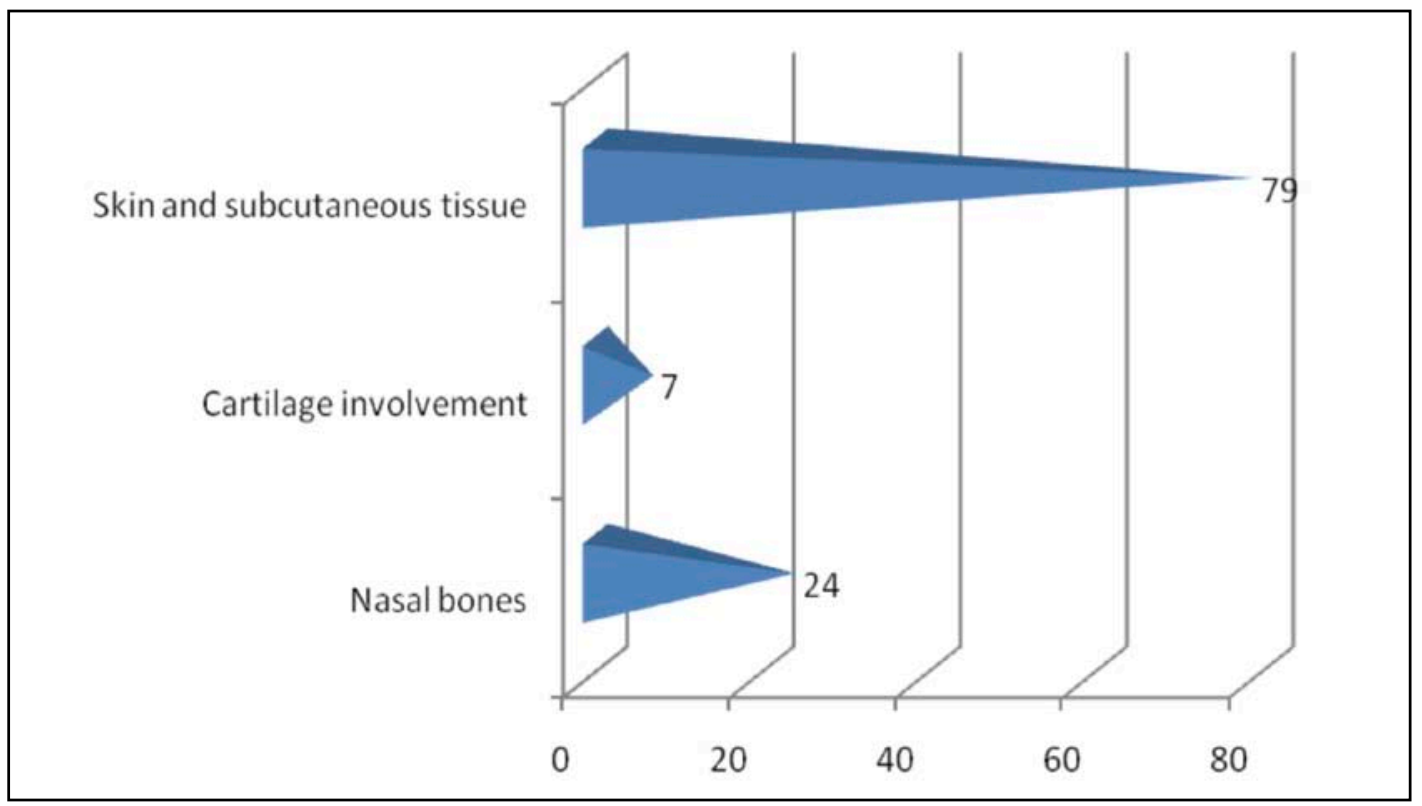

Figure 4. Depth of the defect in the study group

In the patients who presented nasal pyramid fractures (24 patients), the reduction of the fracture was done in 18 cases $(75 \%)$; for 6 of them the manoeuvre was not needed $(25 \%)$.

In $8.18 \%$ of the cases, post-surgery complication occurred (9 patients), while $92 \%$ of the cases were complications free (101 patients) (Figure 7).

$26.36 \%$ of the patients included in the study presented associated diseases (29 patients) and 29.09\% simultaneous wounds (32 patients).
Leukocytes were high in $18.18 \%$ of the patients (20 patients), $9.09 \%$ presented anaemia (10 patients), $9.09 \%$ modification of coagulation times (10 patients) and $3.63 \%$ presented abnormalities of the total proteins (4 patients).

$54.54 \%$ of the patients included in the study were diagnosed with nasal defects secondary to tumor pathology. From them, 26 presented a relapse (Figure 8). 


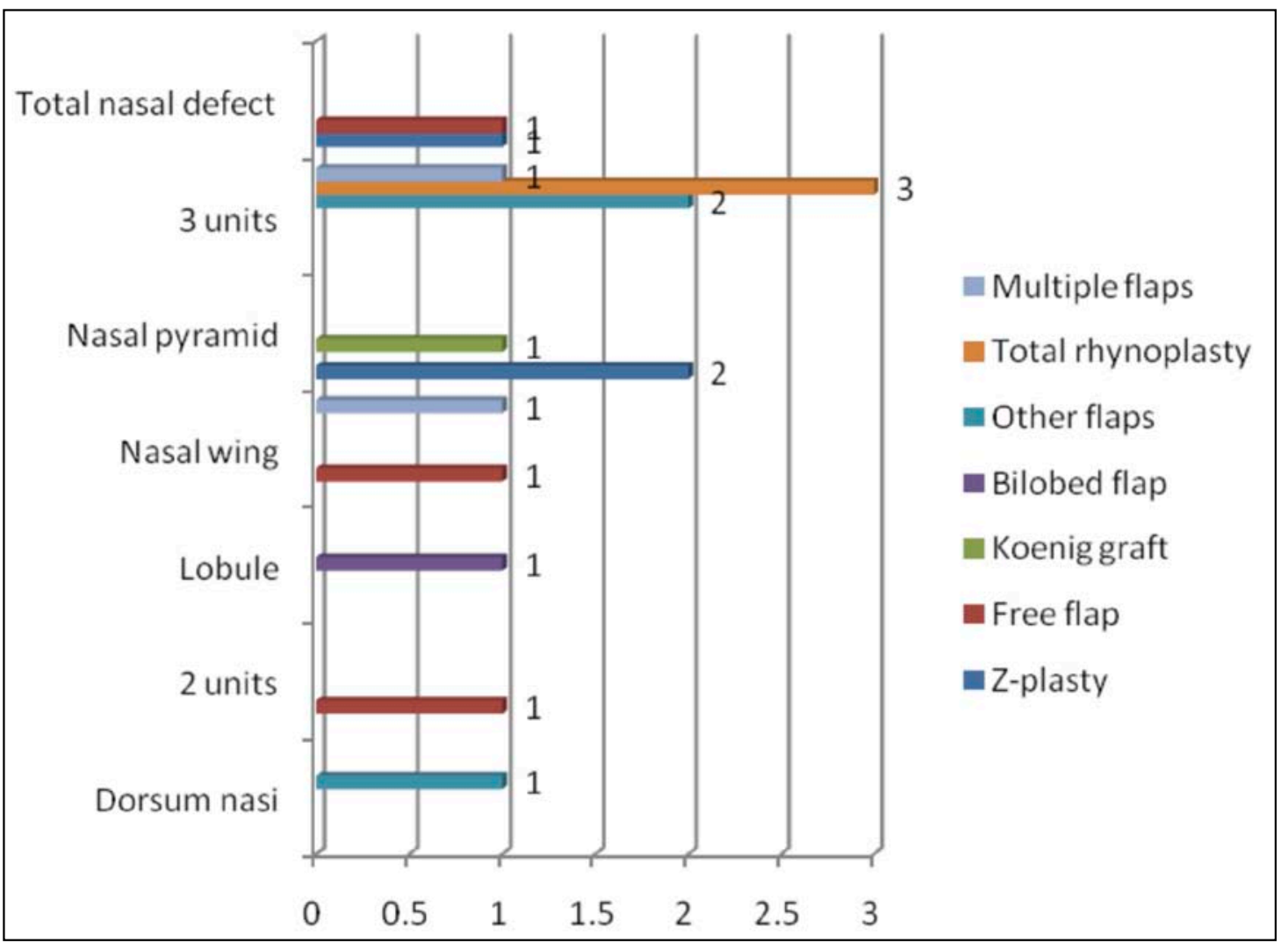

Figure 5. Types of nasal reconstruction regarding the defect topography

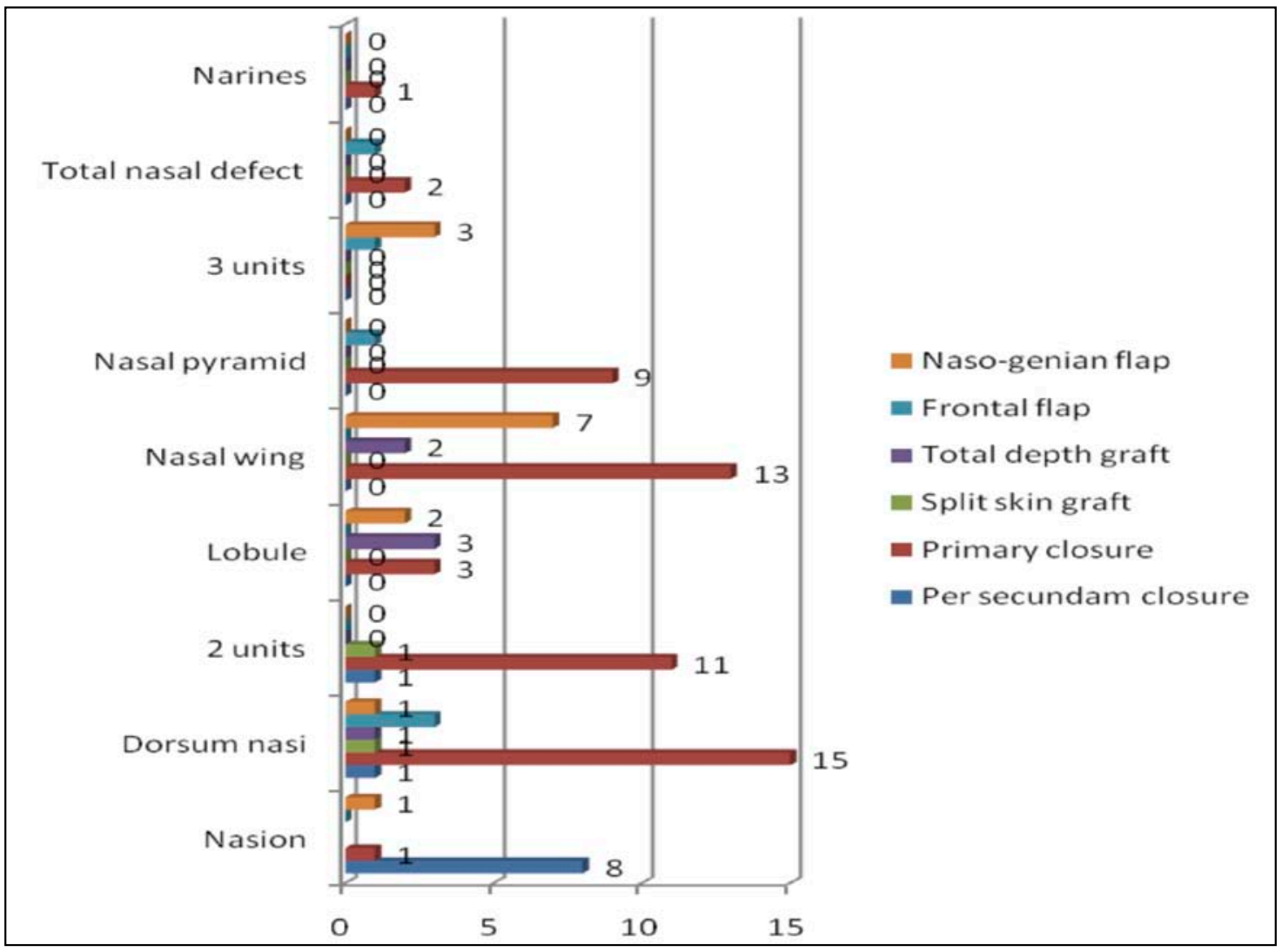

Figure 6. The flaps utilised regarding the topography of the defect 


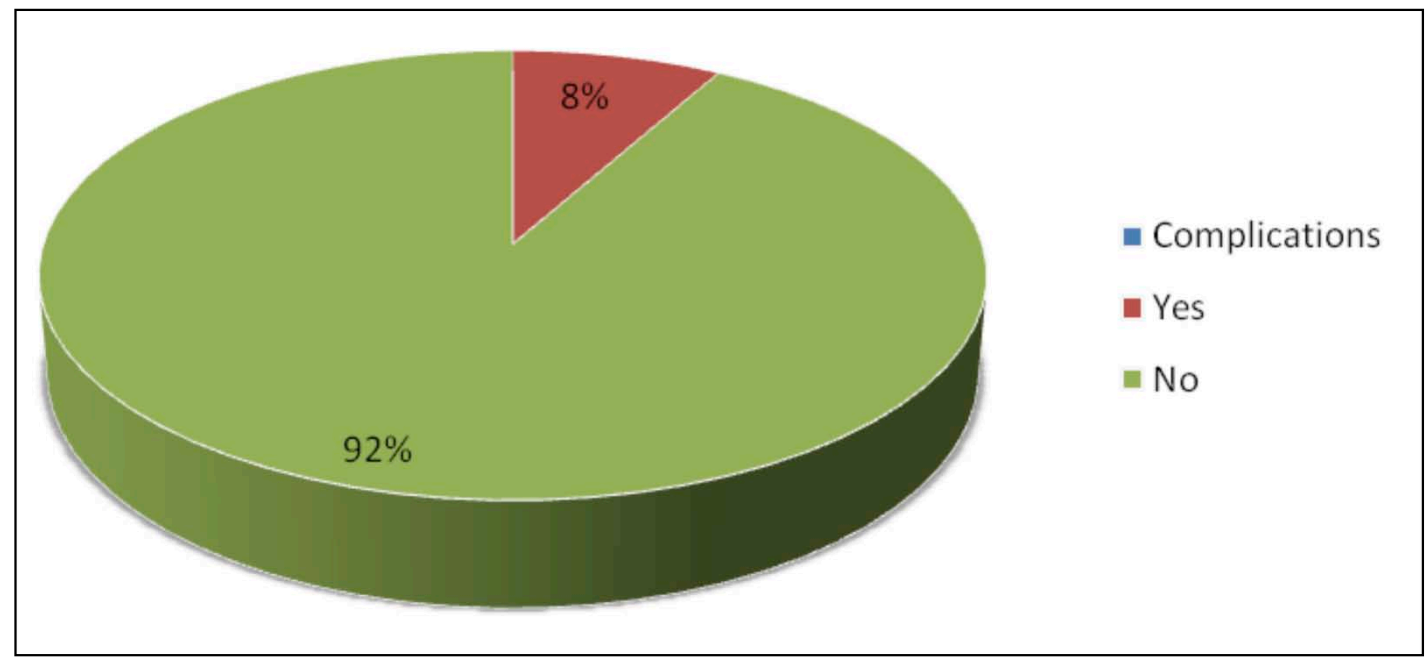

Figure 7. The complication rate after nasal reconstruction using defect reconstruction technique

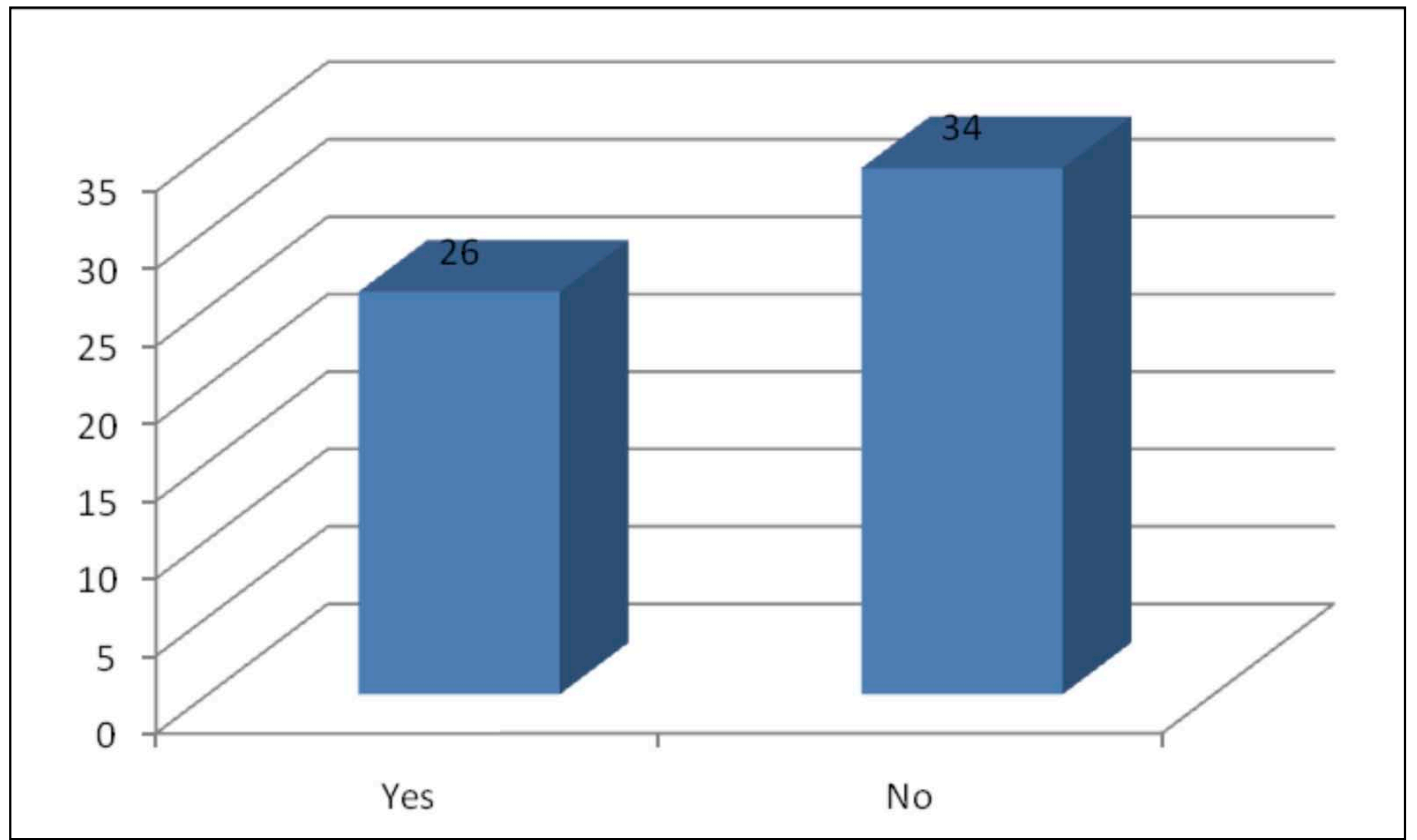

Figure 8. Relapse rate post-excision carcinoma

\section{DISCUSSIONS}

The results of our study regarding the use of the subunit principle in nasal reconstruction showed that good results can be achieved using the defect reconstruction method, sparing healthy tissue, which must be excised in order to respect the nasal subunit principle. Other studies show different results, with good aesthetic and functional outcomes after reconstruction of the whole aesthetic subunits of the nose $\mathrm{e}^{6,7}$.

Despite a large number of studies that discussed between the defect reconstruction and the whole aesthetic subunit, there is no perfect method of nasal reconstruction; both reconstruction types have advantages and disadvantages. The most important aspect is to personalize each case and not to standardize the techniques based only on the nasal subunit principle or on the defect reconstruction method. The nose is a complex anatomical and functional unit of the face, which has an important aesthetic role and must be reconstructed regarding to this conditions, especially in large defects that need in our opinion the defect reconstruction method. This method is supported by other studies; especially in complex defects, skin grafts and local flaps have excellent results ${ }^{8}$.

The free flaps are the reconstruction method used for total nasal defects, but this technique is very difficult and has many complications ${ }^{9}$.

The nasal reconstruction may be done in several steps depending on the complexity of the defect. The 
small defects can be reconstructed using the paramedian flap, which is used on a large scale in plastic surgery $^{10}$. Other local flaps, such as the nasolabial flap, can have excellent results when used for the reconstruction of the nasal wings and the lateral wall ${ }^{11,12}$.

\section{CONCLUSIONS}

The success of the reconstruction of the nose defects depends on the type of defect, its placement and on the simultaneous wounds. The reconstruction type used has a great impact upon the complication or relapse risk and on the number of hospitalisation days.

Although closing per secundam and primary suture were used in many cases, we conclude that the grafts can give very good results in all aesthetic subunits, including the defects that affect three subunits. In this last case, the nasogenian flap and the total rhinoplasty can be techniques of choice.

Also, we used the nasogenian flap with good results for the nasal wing defects and lobule reconstruction. In case of the reconstruction of the dorsum nasi, on the other hand, the frontal flap was used with satisfactory results.

Although in our clinic the reconstruction of the defect is used in principal and rarely the reconstruction based on the aesthetic subunits principle, very good results were obtained, with a reduced number of complications and very few relapses.

Acknowledgement: This paper is supported by the Sectoral Operational Programme Human Resources Development (SOP HRD), financed from the European Social Fund and by the Romanian Government under the contract number POSDRU/159/1.5/137390

\section{REFERENCES}

1. Thornton J.F. - Soft Tissue Facial Reconstruction Guest Editor Nasal Reconstruction: An Overview and Nuances. Semin Plast Surg., 2008;22(4):257-268.

2. Shah A.R., Zoumalan R., M.D., Constantinides M.S. - Aesthetic Repair of Small to Medium-Sized Nasal Defects. Facial Plast Surg., 2008;24:105-119.

3. Haugen T.W., Frodel J.L. - Reconstruction of Complex Nasal Dorsal and Sidewall Defects. Is the Nasal Sidewall Subunit Necessary? Arch Facial Plast Surg., 2011;13(5):343-346.

4. Rohrich R.J., Griffin J.R., Ansari M., Beran S.J., Potter J.K. - Nasal reconstruction-beyond aesthetic subunits: a 15-year review of 1334 cases. Plast Reconstr Surg., 2004;114(6):1405-16; discussion 1417-9.

5. Singh D.J., Bartlett S.P. - Aesthetic considerations in nasal reconstruction and the role of modified nasal subunits. Plast Reconstr Surg., 2003;111(2):639-48; discussion 649-51.

6. Suárez J.E., Sadigh P.L., Jeng S.F. - Aesthetic Subunit Reconstruction Facilitated with V-Y Island Advancement Flaps on the Face: A Case Report. Plastic Reconstr Surg Glob Open, 2014 Jun 6;2(5):e148. doi: 10.1097/ GOX.0000000000000107. eCollection 2014.

7. de Pochat V.D., Alonso N., Ribeiro E.B., Figueiredo B.S., de Magaldi E.N., Cunha M.S., Meneses J.V. - Nasal reconstruction with the paramedian forehead flap using the aesthetic subunits principle. J Craniofac Surg., 2014 Nov;25(6);2070-3. doi: 10.1097/SCS.0000000000001152.

8. Goulão J. - Reconstruction of a large nasal defect. J Dermatolog Treat., 2014 Oct;25(5):444-445. doi:10.3109/09546634.2012.755257. Epub 2013 Feb 3.

9. Moore A.M., Montgomery J., McMahon J., Sheikh S. - Osteocutaneous radial forearm free flap in subtotal nasal reconstruction. BMJ Case Rep., $2014 \mathrm{Nov}$ 26;2014. pii: bcr2014207147. doi:10.1136/bcr-2014-207147.

10. Menick F.J. - Forehead flap: master techniques in otolaryngology-head and neck surgerys Facial Plast Surg., 2014;30(2):131-144. doi: 10.1055/s-0034-1371904. Epub 2014 May 8.

11. Bi H., Xing X., Li J. - Nasolabial-alar crease: a natural line to facilitate transposition of the nasolabial flap for lower nasal reconstruction. Ann Plast Surg., 2014 Nov;73(5):520-4. doi: 10.1097/SAP.0b013e31827f547e.

12. Kang I.G., Jung J.H., Kim S.T., Kim Y.J. - Reconstruction of a columellar defect with a nasolabial island flap. Clin Exp Otorhinolaryngol., 2014 Jun;7(2):142-144. doi: 10.3342/ceo.2014.7.2.142. Epub 2014 May 21. 